\title{
Access to medical records for the purposes of medical audit
}

\author{
STATEMENT BY THE AUDIT WORKING GROUP OF THE CONFERENCE OF MEDICAL ROYAL COLLEGES AND THEIR FACULTIES IN \\ THE UNITED KINGDOM
}

The conference has previously published a statement on confidentiality in relation to medical audit.' The audit working group of the conference has learnt of some concerns about rights of access to medical records for the purposes of medical audit. Some health care professionals have suggested that the consent of the patients concerned should be sought, or approval obtained from the local ethics committee. A previous report by the Royal College of Physicians on the practice of ethics committees in medical research involving human subjects has made some observations which are relevant to these points. ${ }^{2}$

\section{Paragraph 4.8 states:}

"Ethical review is not required for studies that amount to quality control or medical audit, such as 'the aggregation or analysis of information for the purpose of monitoring the provision and effectiveness of health care services or for inquiries designed to establish indices of morbidity or mortality in various fields of practice, provided always that the results of the aggregation or analysis are not made available in a form which identifies the subjects of the information."

In regard to the use of personal medical records for audit purposes, paragraph 11.23 states that:

"There is a duty to use available information for the general good where this can be done without detriment. Use of personal medical records without approaching or involving the patients concerned is, in principle, ethically acceptable provided confidentiality and anonymity are preserved."

It is likely that as the techniques of medical audit improve, data will be collected from the records of patients treated in more than one institution providing health care, not only to increase the numbers available for audit purposes, but also to compare practice and outcomes between providers. In relation to this, paragraph 11.24 states:

"Normally, access to personal medical records for formal protocol based studies should be reviewed by the local research ethics committee. However, ethics committees need not be concerned with work which involves what amounts to quality control, medical audit or preliminary clinical appraisal, including work to establish possible causes of disease. Some studies that are much in the public interest-for example, some nation wide epidemiological studies-may be impeded or even prevented by impracticable requirements-for example, that investigators approach every local research ethics committee and each patient for access to medical records. In such circumstances the investigator should obtain approval from one ethics committee, usually his local committee. The investigator may then write to chairmen of local ethics committees for authorisation to approach directly the custodians of medical records. To expedite progress of research, it may be assumed that permission to approach the custodian directly is agreed if no objection is made within a reasonable time-for example, three weeks. Normal practice regarding access to medical records should be followed. This involves seeking approval of clinicians responsible for the patients as set out in the 'Report of the College Working Party on Research Involving Patients ${ }^{3}$ or, in the case of information abstracted from personal records, the agreement of the custodian of that information."

\section{Pargraph 11.25 states:}

"It may be that in the future such nationwide work will be conveniently reviewed through a central body."

\section{Paragraph 11.26 states:}

"Where patients having a particular condition are identified by scanning registers and it is desired to approach them with a view to research, this should be done via the patients' personal doctors."

The audit working group of the conference supports all these statements cited above.

The audit working group draws attention to one type of audit about which it would be right to obtain the views of the local research ethics committee. Suppose it were decided to audit the quality of information provided to the carers of patients with advanced cancer. There would be difficulties in deciding whether the consent of the patient was required, and questioning might cause distress to the relatives. The auditor might well find it helpful to obtain the views of the local research ethics committee or of its chairman about his or her protocol.

Colleagues may also find it useful to consider the differences between medical practice, medical research, and medical audit. Paragraph 3.1 of the Royal College of Physicians report states:

"In medical practice the sole intention is to benefil the individual patient consulting the clinician, not to gain knowledge of general benefit, though such knowledge may incidentally emerge from the clinical experience gained. In medical research the primary intention is to advance knowledge so that patients in general may benefit; the individual patient may or may not benefit directly."

Medical audit is a review of the structures available for care, the processes of care, and the outcomes of care of a population of patients already treated. The population may be defined by a number of categories such as date on which admitted, firm under the care of which the patient was treated, ICD9 diagnosis, symptom -for example, the management of incontinence, etc. As with any other body of knowledge, there must of necessity be research into the methodology of medical audit.

It is clear that if information is required for the purposes of the research into audit over and above that required for patient care, then the chairman of the local research ethics committee should be consulted. It may be that the additional information required is so straightforward that he or she will advise that a review of the protocol by the full committee is unnecessary. On other occasions, it may be determined that the committee should see a full research protocol.

1 Conference of Medical Royal Colleges and their Faculties in the United Kingdom. Statement on confidentiality and medical audit. BM7 1991;303:1525.

2 Royal College of Physicians. Guidelines on the practice of ethics committees in medical research involving human subject. London: Royal College of Physicians Publications, 1990.

3 Royal College of Physicians. Research involving patients. London: Royal College of Physicians Publications, 1990.
- Mr Nicholas Cheshire will deliver the second Peter Clifford Memorial Lecture on Friday 28 May at St Mary's Hospital during the president's day of the section of surgery, Royal Society of Medicine, $330 \mathrm{pm}$, "Aspects of Molecular Biology and Atherogenesis in Seattle." Details of the lecture, the president's day, and the 1993 Peter Clifford Travelling Scholarship are available from Mr Peter McDonald, consultant surgeon, Northwick Park Hospital, Harrow, Middlesex HAl 3UJ (tel $0818692627)$.

- The Yorkshire Breast Cancer Group is setting up a multicentre prospective study as a result of the studies on the timing of surgery within the menstrual cycle and the outcome of patients with breast cancer. In addition to collecting data on timing of surgery and other interventions such as mammography or cytology, the regularity of cycle, duration and type of contraceptive pill use will also be recorded. Anyone interested in the management of premenopausal breast cancer is encouraged to join the study. Copies of the protocol along with samples of the data collection form may be obtained from the Clinical Trials Unit at the Yorkshire Regional Cancer Organisation, Cookridge Hospital, Hospital Lane, Leeds LS16 6QB (tel 0532 300566).

- The proceeds of a golf tournament at East Sussex National Golf Course, Uckfield, East Sussex, on Thursday 3 June, will go to the David Leng Appeal Fund, which is raising funds for the National Meningitis Trust. The money will be used to support an ultra centrifuge for Southampton University to help in the work on a vaccine for meningococcal meningitis. Several show business and sporting personalities have agreed to play and the organisers, who have set a target figure of $£ 10000$, are looking for sponsors for the event. Donations may be sent to $\mathrm{Mr}$ John Leng, who can provide further information: Woodstock, 2 Buttsfield Lane, East Hoathly, East Sussex.

- SANELINE, the mental health helpline run by the charity SANE, is appealing for volunteers. They do not need to have specific experience in mental illness but they undergo 35 hours of training over five weeks and have continuous inservice training. The helpline is now open every day from $2 \mathrm{pm}$ to midnight and there were nearly 15000 attempted calls between 4 January and 14 February. Anyone interested in becoming a volunteer should ring 0717246570 .
- The National Autistic Society has published a leaflet aimed at people whose work may bring them into contact with children and adults with autism. Single copies of In Your Working Life You Can Expect to Meet Several People with Autism. Will You Recognise Them? is available from the National Autistic Society, 276 Willesden Lane, London NW2 5RB, on receipt of a stamped addressed envelope.

- The Freud Museum, 20 Maresfield Gardens, London NW3 5SX was the home of the pioneer of child psychoanalysis, Anna Freud, wh wanted her home turned into memorial to her father, Sigmund Freud. Now the museum contains a new photographic display to celebrate the life and work of Anna Freud. It is open Wednesday to Sunday, 12 noon to $500 \mathrm{pm}$. 